concentration of the nutrient in the field of a different order from those in the sand cultures described, but also rates at which nutrients in the soil become available are likely to be more important than actual concentrations in the soil solution, and the supply may continue at a relatively high level throughout the growing period. This is specially important in those types of plant, such as barley, in which nutrients taken up after the stage when further elaboration of meristems ceases lead to no further increase in yield but merely raise the concentration within the plant, thereby checking the normal tendency to decline to a minimum at harvest. Hence plant analyses from field crops can show considerable variations in concentration at harvest. High concentration of nutrient in the mature cereal plant may be due either to limitation of growth by some other nutrient factor leading to 'luxury consumption' or to a high supplying power of the soil maintained throughout the growing period. In the former case, the authors point out that yield will tend to be low and could be improved by the addition of the nutrient in minimum; but in the latter case yield will be high. Hence the use of analyses of mature cereal plants as indicators of the nutritional status of soils depends on the fact that differences in the nutrient-supplying power of the soil persist throughout the growing season, leading to accumulation at harvest, and not upon any causal relationship between final internal nutrient concentration and yield.

A good deal of consideration is given to the best method of selecting plants for analysis, to the influence of time of day and time of year or stage of development of the plant when samples are taken, and to the advantages of analysing the whole plant as compared with a single organ of the plant. On the subject of which organ is likely to prove the most suitable for analysis, the authors have collected all the available evidence and present it in tabular form under the various crops. In the majority of cases the leaves seem to show the greatest proportional increase in internal concentration of a nutrient with increase in its supply. Necessary precautions in the preparation of material for analysis are discussed, followed by consideration of the analysis itself, including the need to determine particular chemical fractions and the choice of extraction methods.

The interpretation of the results of diagnostic analysis is perhaps the most difficult part of the whole problem of the use of plant analysis to determ. ine fertilizer needs. Standards of reference are essential, together with data on the performance of the plants from which they are derived. To establish standard values for internal nutrient content requires data on the yield responses of plants at varying levels of nutrition and on their composition. Emphasis is placed on the dangers of basing conclusions on data for a single element without allowing for variations in the content of other nutrients. Nevertheless, it is pointed out that many investigators have specified optimal levels or limiting values above which the supply of a particular nutrient may be regarded as adequate, presumably where the content of other nutrients is not limiting. A comprehensive table of these suggested standard values for nutrient content of a wide variety of crops is included in the paper.

Suggestions by numerous workers for standard ratios of various plant nutrients are also given in tabular form; but the authors state that in their opinion there is no reason for supposing that ratios of nutrients in general are likely to be of greater use in diagnostic work than the content of the elements individually. They consider that ratios of nutrients within the plant should generally be used in conjunction with data for individual concentrations. The authors express the view that 'foliar diagnosis' work must be subject to much the same criticisms as other work involving the use of ratios.

In their final consideration of the place of plant analysis among other methods of nutritional diagnosis, the authors suggest that a combination of soil and plant analyses may give the most satisfactory results : soil analysis to determine pre-planting fertilizer treatment, and plant analysis for perennial crops and to indicate the need of annual crops for side or top dressings of fertilizer. Diagnosis by visual symptoms is an easy and rapid method where symptoms can be readily and definitely recognized, but has the disad. vantage that waiting for deficiency symptoms to appear before treatment can be given may reduce the final yield.

\section{SOCIOLOGY OF MENTAL DISEASE}

THE physical and biological sciences have at last

been accepted as indispensable adjuncts to the art of government, but the psychological and sociological lag behind. No clearer evidence of this is needed than the assumption that the men of science whose help should be asked to solve the problem of controlling atomic bombs are those who are specialists in making the machine rather than those who are specialists in the study of the minds likely to set the machine in motion.

It is encouraging, therefore, that the National Association for Mental Health should have had full and practical governmental support for its conference in the Seymour Hall during January 15-16. By sanctioning the payment of expenses, the various Ministries secured a very large attendance of delegates from local authorities. Moreover, the Parliamentary Secretary to the Minister of Health eontributed that rarest of things - an opening address which must have usefully coloured the minds of everybody throughout the ensuing meetings.

Mr. Edwards emphasized that mental illness is a social disease with sequelæ deleterious to national and international life, that its consequences affect production drives, foreign policies, the family institution, and the efficacy of education. Moreover, it is a matter which cannot be left simply to technicians, since it affects every individual, however sane potentially, since complete sanity is impossible for anyone who has to live in a community riddled with mental and nervous troubles.

Dr. G. R. Hargreaves, principal medical officer of Lever Bros. and Unilever, Ltd., and Dr. Donald Stewart, medical adviser to the Austin Motor Co., provided the text for the conference by epitomizing the Russell Fraser Report on the incidence of neurosis among factory workers*. In a sample of more than three thousand male and female workers in light and medium engineering factories, 10 per cent suffered from definite and disabling neurotic illness and a further 20 per cent from minor forms of neurosis during the course of six months. Neurotic illness accounted for a loss of three working days by every man studied and six days by every woman. It is at least as important a source of industrial loss as colds and influenza, or accidents and industrial disease.

* No. 90. Industrial Health Research Board, 1947. (H.M. Stationery Office.) $18.3 d$. 
Moreover, since neurosis is a disease of human relationships, in which the sick man's associates and family suffer, there must be added an incalculable loss caused by interference with the smooth working of those who do not themselves report sick.

Dr. Hargreaves suggested that hitherto social effort has been concentrated on psychotics and serious certifiable disease too exclusively, and that so long as the whole problem outruns the supply of trained personnel it is wiser to concentrate on the lighter neurotic diseases which interfere far more with the social machine than the psychotic do.

Dr. Kenneth Soddy, medical director of the National Association for Mental Health, and Dr. Doris M. Odlum, vice-president, discussed the obligations and opportunities given to local authorities by the National Health Service Act, 1946, in dealing with this problem.

Dr. Soddy gave a series of case-histories which showed that the problem of the neurotic cannot be treated as a purely medical one, and that the psychiatric social worker has a specific task, involving the mastering of a difficult technique, to co-ordinate the various administrative organs which might be needed to cope with a socially and personally 'unstuck' individual.

It is clear that the provision of sufficient trained psychiatric social workers-one in 2,500 of the population-is virtually impossible for many years. Not only is it impossible to train this number; but also there are probably not sufficient people of the right character and temperament fit for training. It is on this problem that it was possible to sense a difference of opinion within the conference.

On one hand, Dr. Greenwood Wilson, medical officer of health at Cardiff, speaking as a delegate, said that Cardiff proposes to use health visitors to help supplement the trained psychiatric workers, and pointed out that health visitors are not only experienced in human nature, but also masters of the special technique of breaking into the family circle in quest of private and intimate information. On the other hand, Brigadier A. Torrie, director of army psychiatry, gave his opinion that only a person with psychiatric training could be entrusted with any part of the work. It is to be hoped that Brigadier Torrie is wrong, since we shall not have a hundredth part of the required army of psychiatric workers trained for years to come. Fortunately, the National Association for Mental Health is aware of the danger of establishing a vested interest point of view on this matter.

The real difficulty is that applied psychiatry involves two requisites: a technique to be acquired by a specific training, and a personal character which cannot as yet be acquired. An industrial physicist or chemist may be a drunkard, a wife-beater or a child murderer without it necessarily interfering with his scientific work; but an industrial psychiatrist can ill afford these idiosyncrasies.

Whatever it means to-day, psychology used to mean the study of the human 'soul', and there used to be a profession or a vocation called a 'cure of souls'. Why the cure of souls has been taken over from the clergyman by the psychiatrist is a question which cannot be gone into here. Possibly there are too many clergy who have character without technique, but it is certain that technique by itself will be no adequate substitute for character. The admirable speech of the Earl of Feversham, chairman of the National Association, left the conference in no doubt that the Association is fully alive to this necessity for wedding technique and character in all cure of souls, and along with Mr. Edwards' opening address must have sent the delegates away realizing that they must act not only as administrators but also as human beings if the rising tide of mental sickness is to be held back. Both Dr. Odlum and Miss Clare Britton repeatedly emphasized that good results depended on personnel who know how to be humane. Miss Britton made a good point when she suggested that personnel for boarding schools for difficult children should never be expected to spend more than seven years of their lives at this exacting task. All psychiatry involves such inroads on emotional resources, apart altogether from its demands on those parts of the human intellect usually called upon for scientific work, that it should only be practised for limited periods.

It is to be hoped that all local authorities represented at the conference will have been impressed with the opportunities afforded them under the new Act for dealing with the chronic minor diseases of human relationships which cause so much more trouble than the far rarer and more spectacular mental disorders to which their attention was once confined.

\section{ASSOCIATION OF BRITISH ZOOLOGISTS}

$\mathrm{D}^{\mathrm{s}}$ ESPITE the predominantly urban nature of the population of Great Britain, one of its characteristics at the present time is a reawakened interest in, and a feeling of responsibility for, the countryside. This is made more acute by the realization of the part which agricultural workers can play in supplying food, and by the fear that, in a small island like Great Britain, indiscriminate exploitation by industry or the Services might destroy what could never be replaced. That this spirit is beginning to permeate the teaching of biological subjects in both schools and universities was indicated at the meeting of the Association of British Zoologists held on January 8, in the rooms of the Zoological Society of London. Prof. H. Graham Cannon was in the chair and the general topic of discussion was "The Field Study and Conservation of the British Fauna".

The universities of Britain are, for the most part, situated in its big towns, and at present the combined difficulties of congested time-tables, large classes and inadequate staff often mean that little or no fieldwork can be introduced into their zoological courses. Such work of this type as has been done in the past has almost invariably been marine, and the field courses of longest standing are those held at the various marine laboratories. These, as was stressed by Prof. F. W. Rogers Brambell, must remain the most important in the training of the general zoologist; but they should be supplemented by others which would introduce the student to freshwater or entomological ecological problems, and which would provide special training in these where that is necessary. Prof. J. W. Munro described in this connexion the way in which the Silwood Park Field Station of the Imperial College of Science and Technology could be used for both undergraduate teaching and for introducing postgraduate workers to field research in entomologya very important development if many of the schemes for the Colonies, such as that for growing groundnuts in Africa, are to be supplied with properly trained biologists. Without the help of such men, all these 